\title{
Wobbling of a liquid column between unequal discs
}

\author{
I. Martínez , J. Meseguer, J.M. Perales \\ IDRIUPM, Universidad Politécnica de Madrid, $P_{\bar{z}}$. Cardenal Cisneros sin (cto ETSIA), 28040 Madrid, Spain
}

\begin{abstract}
One of the most puzzling results of an experiment on the stability of long liquid columns under microgravity, performed aboard Spacelab-D2 in 1993 and named STACO, aiming at the analysis of deformations of nearly cylindrical liquid columns under several mechanical disturbances, is revisited here. It corresponds to the unexplained breakage of an $85 \mathrm{~mm}$ long liquid bridge of low viscosity silicone oil, established between unequal discs of 30 and $28 \mathrm{~mm}$, intended to counterbalance the expected deformation by residual acceleration found in previous flights, and left idle because the vibrations and oscillations to be applied afterwards were not started, for fear of premature breakage. A detailed image analysis is performed to extract the maximum amount of data, to be able to check against available theories for axisymmetric and non-axisymmetric deformations of a liquid column.
\end{abstract}

Keywords: Spacelab; Microgravity; Experiment; Liquid bridge; Capillarity; Fluid mechanics

\section{Introduction}

A series of experiments with long liquid columns, typically $85 \mathrm{~mm}$ long by $30 \mathrm{~mm}$ in diameter, has been carricd out on space platforms (orbiting Spacelab and Texus sounding rocket), using the liquid shape as a detector of controlled and uncontrolled mechanical disturbances acting upon the liquid, to verify that the static and dynamic models of the behaviour of the liquid column are relevant in these microgravity conditions, unavailable on ground. Besides, the liquid column configuration provides a fluid-mechanical model of the molten bridge in the floating-zone technique of crystal growth and high melting point refining in material sciences, and may be used to measure interfacial tension and other propertics of molten materials.

Liquid bridges are formed when a finite volume of liquid is established between two or more non-contacting solids. Size is important in practice, and there are great differences from submillimetric liquid bridges that can be manipulated under the microscope, and centimetric liquid bridges in a space platform, if only for their response time: from a few hundredths of a second to more than $10 \mathrm{~s}$, respectively. The basic liquid bridge (the one with the simplest geometry) is a cylindrical liquid mass spanning between two equal and coaxial circular dises in weightlessness, the liquid being held together by capillary forces, anchored to the edges of the solid discs, and surrounded by air; what is called a liquid column. A summary of liquid bridge research can be found in Martínez and Perales (2001).

Unfortunately, a few hours of experimental time in a totally new laboratory environment in an unfriendly operational scenario with scarce interactivity, non-transparent voice loops, ficld work for the scientists, crowded timeline for the crew on board, with new facilitics that cannot be tested before flight, has proved insufficient for a satisfactory explanation of several important results, such as the unexpected breakage aboard Spacelab-D2 in 1993, revisited here, the non-uniform residual acceleration level found on Spacelab-D1 and Spacelab-D2, or the loss of anchorage on breaking of the liquid to its supports found on Spacelab-D2 (in 
contrast with the perfect anchoring in Spacelab-Dl). Fortunately, many other problems found earlier have been solved. We learned to establish large liquid columns between two protruding solid dises, by filling liquid through a hole in one disc, either with the discs some distance apart, or by separating the dises while filling. We have mastered the visualisation and image recording process that caused so many problems in the past. We have verified that the average shape of the liquid corresponds to the theoretical equilibrium shapes, that dynamic shape deformation matches their theoretical eigenfunction; that the natural frequency of free oscillations coincides with theoretical prediction, that the vibration pattern is as predicted by theory, and so on.

The aim of this paper is to perform a refined analysis of a unique experimental result obtained in SpacelabD2. This was the third run on the same flight of an experiment named STACO, and nothing similar happened in the two previous trials, although a consistent tendency for the liquid to be slightly pushed towards the feeding disc could be observed on the three runs. An effort has been made to keep the description to the 'as flown' experiment, avoiding all the intricate changes to the nominal timeline that were introduced during the flight (because of lack of time, lack of video and voice contact, hardware malfunction or spot opportunities). A different approach to our previous publication (Martínez et al., 1995) is also followed here for the im- age analysis, based now on automated edging of the whole digitised image sequence (from the old VHS master copy, already converted from NTSC to PAL format), instead of being based on just one-line on-theflight scanning from the video play. Two video-frames from the experiment are shown in Fig. 1, with their automatically extracted outlines, where an S-shaped nearly axisymmetric liquid column at the centre can be seen against a diffusively retro-illuminated background grid with some vertical lines and squares (and a time stamp) to help in the image analysis (e.g., the first image was shot at Greenwich-Meridian-Time on the 125th day, $14 \mathrm{~h}, 15 \mathrm{~min}, 35 \mathrm{~s}$, of year 1993 ).

\section{Theoretical model}

It is known that, in perfect weightlessness, the stability of a cylindrical liquid column of length $L$ and radius $R_{0}$ is bounded by the Plateau-Rayleigh limit for the column slenderness $A=L /\left(2 R_{0}\right)<\pi$. Besides the slenderness, two other geometrical parameters appear in this experiment: the volume of liquid relative to that of the cylinder, $V=V /\left(\pi R_{0}^{2} L\right)$, and the relative difference in radii, $h=\left(R_{2}-R_{1}\right) /\left(R_{2}+R_{1}\right), R_{1}$ being the smaller radius. We are only considering small deformations of the liquid shape relative to that of a cylinder with a mean radius $R_{0}=\left(R_{2}+R_{1}\right) / 2$, near its stability limit,
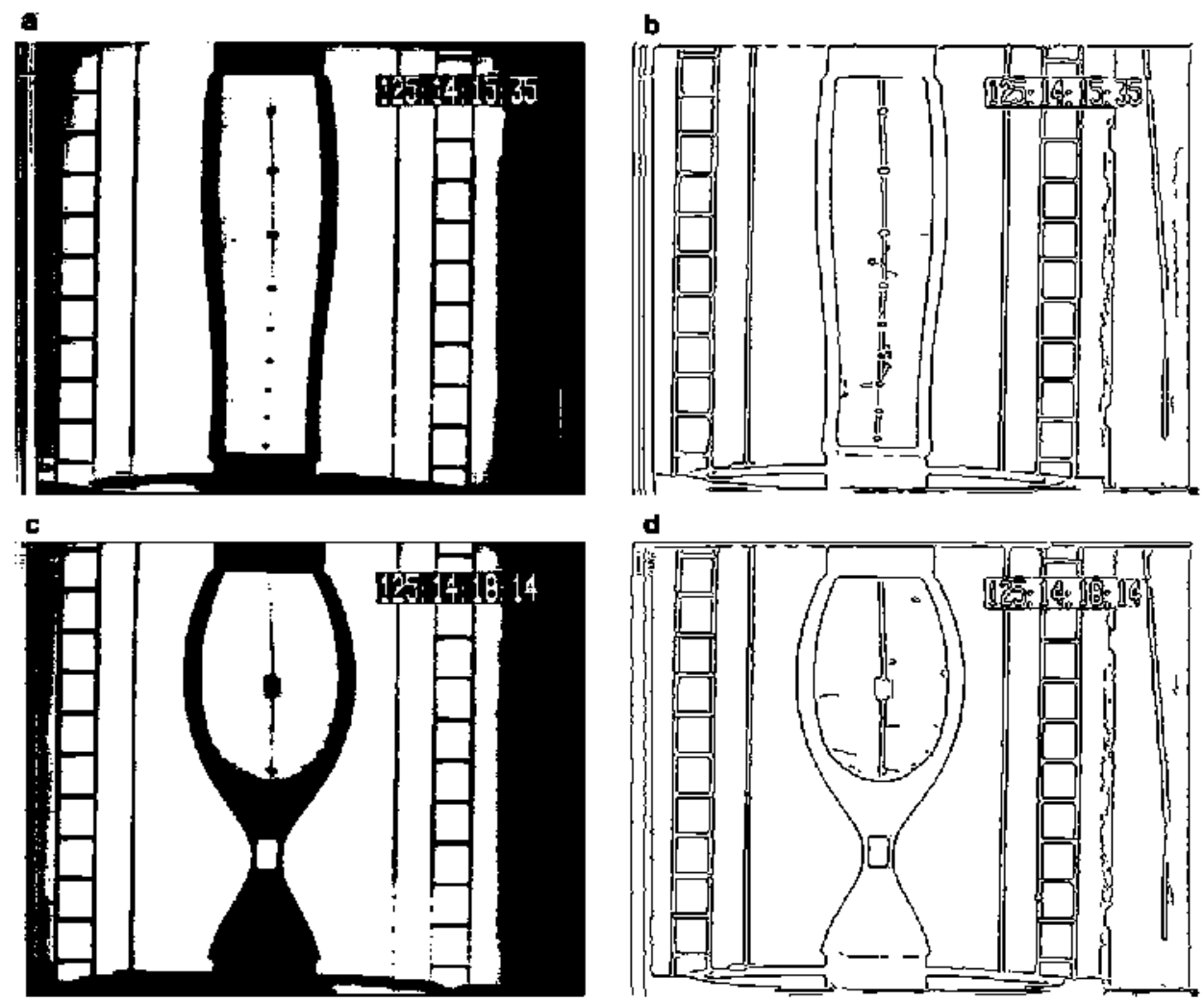

Fig. 1. Video-frames from the beginning and end of the experiment, and their edges autonatically extracted. 
$A=\pi$, so that we adopt as control parameters the following small non-dimensional values: $\lambda=\Lambda / \pi-1, v=$ $V-1$, and $h$. In our case, they have the values $\lambda=-0.067 \pm 0.01, v=0.125 \pm 0.01$, and $h=0.035 \pm$ 0.001 , corresponding to $L=85 \mathrm{~mm}, R_{1}=14 \mathrm{~mm}, R_{2}=$ $15 \mathrm{~mm}\left(R_{0}=14.5 \mathrm{~mm}\right)$, and $V=63.2 \mathrm{~cm}^{3}$.

Besides geometry, the following additional static parameters are of interest: a residual possible axial acceleration, $g_{\mathrm{A}}$, made dimensional as $B o_{\mathrm{A}}=\rho g_{\mathrm{A}} R_{0}^{2} / \sigma$, where $B o_{\mathrm{A}}$ is the axial Bond number, a similar lateral acceleration, $g_{\mathrm{L}}$, made dimensional as $B o_{\mathrm{L}}=\rho g_{\mathrm{L}} R_{0}^{2} / \sigma$, where $B o_{\mathrm{L}}$ is the lateral Bond number, and a possible centripetal acceleration due to a solid-body rotation $\Omega$, made dimensional as $W e=\rho \Omega^{2} R_{0}^{3} / \sigma$, where $W e$ is the Weber number. Notice that there are only two scale factors to pass from non-dimensional values to dimensional ones: the length scale $R_{0}=14.5 \mathrm{~mm}$, and the time scale $t_{0}=\sqrt{\rho R_{0}^{z} / \sigma}=0.35 \mathrm{~s}$.

The equilibrium shape in non-dimensional cylindrical coordinates, $r(z, \theta)$, for a quasi-cylindrical liquid column $(r-1 \ll 1)$, can be analytically expressed as (Meseguer et al., 1996)

$$
\begin{aligned}
r(z, \theta)= & 1+h \frac{\sin z}{\sin A}+v \frac{A(\cos A-\cos z)}{2(A \cos A-\sin A)} \\
& +B o_{\mathrm{A}}\left(z-A \frac{\sin z}{\sin A}\right)+B o_{\mathrm{L}} \frac{\left(A^{2}-z^{2}\right)}{2} \\
& \times \cos \theta
\end{aligned}
$$

where the origin has been taken centred in the middle of column, so that the smaller dise is $r(-A)=1-h$ and the larger disc $r(+A)=1+h$. Eq. (1) is valid in the linear limit even for $W^{e} \neq 0$ (isorotation does not affect the shape of a quasi-cylindrical liquid column, to a first approximation). Two important corollaries follow from Eq. (1):

- A small disc-size difference, measured by $h$, causes a large radial deformation, measured by the radial deformation at a quarter of the column length, $a=r(\Lambda / 2)$. The amplification is deduced from $\mathrm{Eq}$. (1) to be $a / h=1 / \sin A=4.8$ for our case $(A=85 /$ $29=2.93$ ). That means that a $0.5 \mathrm{~mm}$ of disc-radii difference relative to the $14.5 \mathrm{~mm}$ of the mean radius in our case, gives rise to a bulge of $2.4 \mathrm{~mm}$ at a quarter of the column length.

- A given residual acceleration causes a larger radial deformation if it acts axially than if it acts laterally (for slender columns, as here). The amplification can be easily deduced from $\mathrm{Eq}$. (1) and, in our case of $A=2.93$, are $r(A / 2) / B o_{\mathrm{A}}=12.5$ and $r(0) /$ $B o_{\mathrm{L}}=4.3$ (notice that $B o_{\mathrm{A}}$ causes the larger radial deformation at $z=A / 2$, whereas $B o_{\mathrm{L}}$ causes the larger radial deformation at $z=0$ ).

But not every equilibrium shape given by Eq. (1) is feasible. For a liquid column to be stable, the following inequation must be satisfied by the controlling parameters:

$-\lambda+\frac{v}{2}-\left(\frac{3}{2}\right)^{4 / 3}\left(\frac{h}{\pi}-B o_{\mathrm{A}}\right)^{2 / 3}-\frac{\pi^{2}}{4} B o_{\mathrm{L}}^{2}-\frac{W e}{2}-K(t)>0$,

where a qualitative kinetic term $K(t)$ has been added to the stability limits deduced in Martínez et al. (1992) just to pinpoint that any motion would decrease this linear static stability margin. The stability criterion in Eq. (2) have the following implications:

1. For an static unloaded liquid column $(K(t)=W e=$ $B o_{\mathrm{A}}=B o_{\mathrm{L}}=0$ ), the stability criterion reduces to $-\lambda+t / 2-(9 h /(4 \pi))^{2 / 3}>0$. Substituting for this experiment with $\lambda=-0.067, v=0.125$, and $h=$ 0.035 , gives a certain stability margin to cope with possible g-jitter motions or any other mechanical noise.

2. An increase in $K, W e, B o_{\mathrm{L}}, B o_{\mathrm{A}}$, or $h$ destabilises the column, but notice that the effects of $h$ and $B o_{\mathrm{A}}$ might compensate (if the axial acceleration is towards the smaller disc).

3. An increase in $v$ (barrel shape) stabilises the column.

The original objective of this run with unequal discs was to counterbalance the apparent constant residual axial acceleration detected in Spacelab-D1, some $g_{\mathrm{A}}=$ $0.7 \times 10^{-3} \mathrm{~m} / \mathrm{s}^{2}(70 \pm 10 \mu \mathrm{g}$, equivalent here to a value $B o_{\mathrm{A}}=0.007$ ) pushing against the feeding disc in the FPM (here the lower disc), too large a value to be explained by residual air drag and other orbital-mechanics effects from the Shuttle, which can only account for less than $1 \mu \mathrm{g}$. According to Eq. (2), a value of $h=\pi B o_{\mathrm{A}}=$ 0.022 would exactly balance this apparent residual acceleration, corresponding to a difference in disc diameter a little greater than $1 \mathrm{~mm}$. The values finally selected by the investigators were $R_{1}=14 \mathrm{~mm}$ and $R_{2}=15 \mathrm{~mm}$, what would over-compensate the expected acceleration. A mean cylindrical volume, $v=0$, was foreseen so that if the astronaut were able to build the column with $\lambda=-0.067, v=0$, and $h=0.035$, it would mean that the wanted axial acceleration had stabilised the column, otherwise $-\lambda+v / 2-(9 h /(4 \pi))^{2 / 3}=-0.02<0$. It is not a surprise that the operator-astronaut decided to increase the volume of liquid over the nominal procedures, realising that the column was loosing stability while trying to reach the full length. The stability region in the $B o_{\mathrm{A}}-v$ plane for $\lambda=-0.067$ and $h=0.035$ (our case) is presented in Fig. 2 and compared with the stability region for the two previous STACO runs.

The key question now is that, once a stable configuration was reached with $\lambda=-0.067, v=0.125$, and $h=0.035$, and it was left idle, without any detectable disturbance acting upon, what might have happened for the liquid column to gently oscillate for nearly 3 min without decaying, and then break apart? 


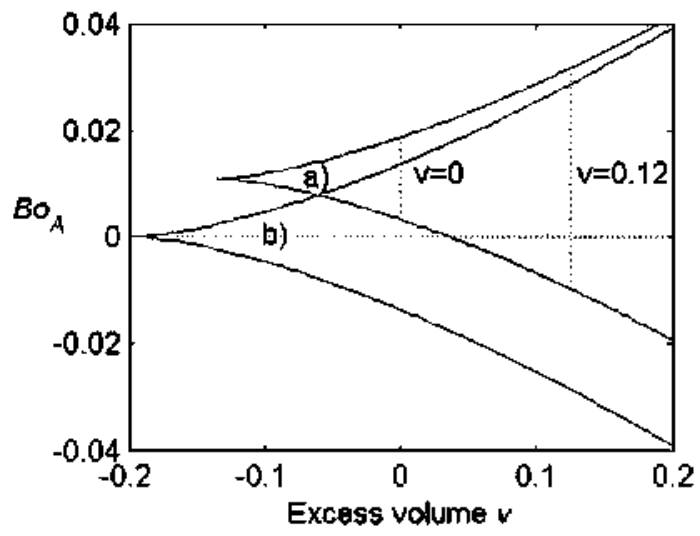

Fig. 2. Stability margin in terms of excess volume, $v$. and axial acceleration, $B o_{\mathrm{A}}$ : (a) for run 3 of STACO $(v=0.12 . h=0.035$, $\lambda=-0.067)$; (b) for runs 1 and 2 of STACO (v $=0, h=0, \lambda=-0.1$ ). The stable region in each case is the region between the curves.

\subsection{The dynamic problem}

One of the bitter lessons learnt from our space experiments with liquid columns near there stability limit has been that dynamic effects are unavoidable. The reason is that we are still unfamiliar with the extremely low forces that shape large liquid masses under microgravity, and which cannot be overridden in an actual flight: the socalled g-jitter, which in combination with an easily moving low-dissipative fluid mass, makes the liquid wobble all the time.

There are several external loads that can force the liquid to move; here, we consider only two forces: an axial component of an unknown acceleration slowly varying with time, $B o_{\mathrm{A}}(t)$, or a lateral component of an unknown acceleration slowly varying with time, $B o_{\mathrm{L}}(t)$, acting upon a liquid column of constant liquid volume between given unequal discs at a constant separation.

For the axial dynamics of long liquid bridges, we model small oscillations around the equilibrium shape by:

$r(z, t)=r_{\text {¿q }}(z)+a(t) \sin \frac{\pi z}{A}$

where the equilibrium shape, $r_{\mathrm{eq}}(z)$, is given by $\mathrm{Eq}$. (1) with all parameters there having constant values, and $a(t)$ is the radial departure from equilibrium at a quarter
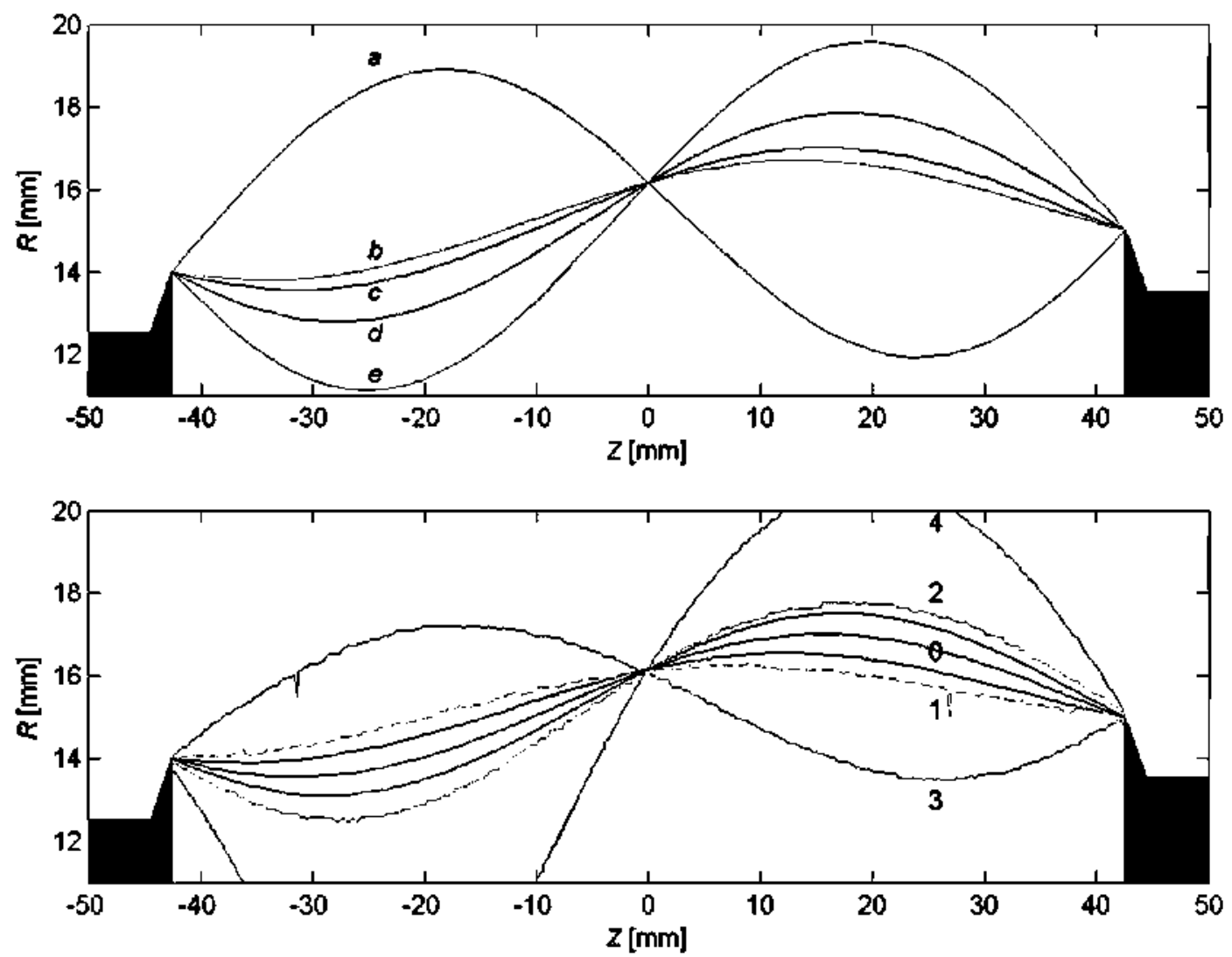

Fig. 3. Upper: some expected axisymmetric equilibrium shapes computed from Eq. (1) with $\lambda=-0.067, v=0.125$ and $B o_{\mathrm{L}}=0$ : (a) maximum stable axial acceleration pushing the liquid against the smaller disc $\left(B o_{\mathrm{A}}=0.032\right)$ : (b) foreseen acceleration from SL-D1 experience $\left(B o_{\mathrm{A}}=0.007\right)$ : (c) acceleration corresponding to the experimental mean shape average from the $160 \mathrm{~s}\left(B o_{\mathrm{A}}=0.005\right)$ : (d) no acceleration $\left(B o_{\mathrm{A}}=0\right)$; (e) maximum stable axial acceleration pushing the liquid against the larger disc $\left(B o_{A}=-0.0097 \approx-0.010\right)$. Lower: some actual shapes during the experiment (the chronological order is $0,1,2,3,4)$, where 0 is mean shape (the liquid outline oscillates around this average shape within the shaded region during the first $120 \mathrm{~s}$ ). (1) first local extreme in radial deformation during the breaking $(t=134.3 \mathrm{~s})$, (2) second local extreme $(t=140.7 \mathrm{~s})$, (3) third local extreme $(t=149.5 \mathrm{~s})$, and (4) shape at breaking $(t=159.1 \mathrm{~s})$. Notice that radial deformations are magnified. 
of the column length, $z=A / 2$, the first eigenfunction of the dynamic problem being $\sin (\pi z / \Lambda)$ (antisymmetric, with a node midway between the discs). One of the best available axial-dynamic model is from Meseguer et al. (1994), which has been recently applied with great success to our sister experiment on Texus-33 (Martínez et al., 2004)

$m \frac{\mathrm{d}^{2} a(t)}{\mathrm{d} t^{2}}+m C \frac{\mathrm{d} a(t)}{\mathrm{d} t}+\lambda a(t)+\frac{3}{4} a(t)^{3}=B o_{\mathrm{A}}(t)$,

where $m=25 / 8$ is an analytical non-dimensional inertiaterm coefficient (deduced considering the simplified inner velocity field given by the so-called Cosserat model) and $C=O h^{1 / 2}=v(\rho /(\sigma R))^{1 / 2}=0.0175$ the square root of the Ohnesorge number accounting for viscous dissipation (with $v=5 \times 10^{-6} \mathrm{~m}^{2} / \mathrm{s}, \rho=920 \mathrm{~kg} / \mathrm{m}^{3}, \sigma=0.019 \mathrm{~N} / \mathrm{m}$, $R_{0}=0.0145 \mathrm{~m}$ ).

We have run this dynamic model in two canonical cases of loading: a step in axial acceleration, and a harmonic excitation with the apparent response period from the images, $T=12 \pm 0.2 \mathrm{~s}$, that is very close with the foreseen natural frequency of the liquid column, given by Eq. (4), $T / t_{0}=2 \pi \sqrt{m /(-\lambda+v / 2)}=31$, or multiplying by the scale $\left(t_{0}=0.3475 \mathrm{~s}\right) T=10.7 \mathrm{~s}$. The response of the liquid to this two simulated loads is shown in Fig. 4. The step value, $B o_{\mathrm{A}}=0.005$, has been
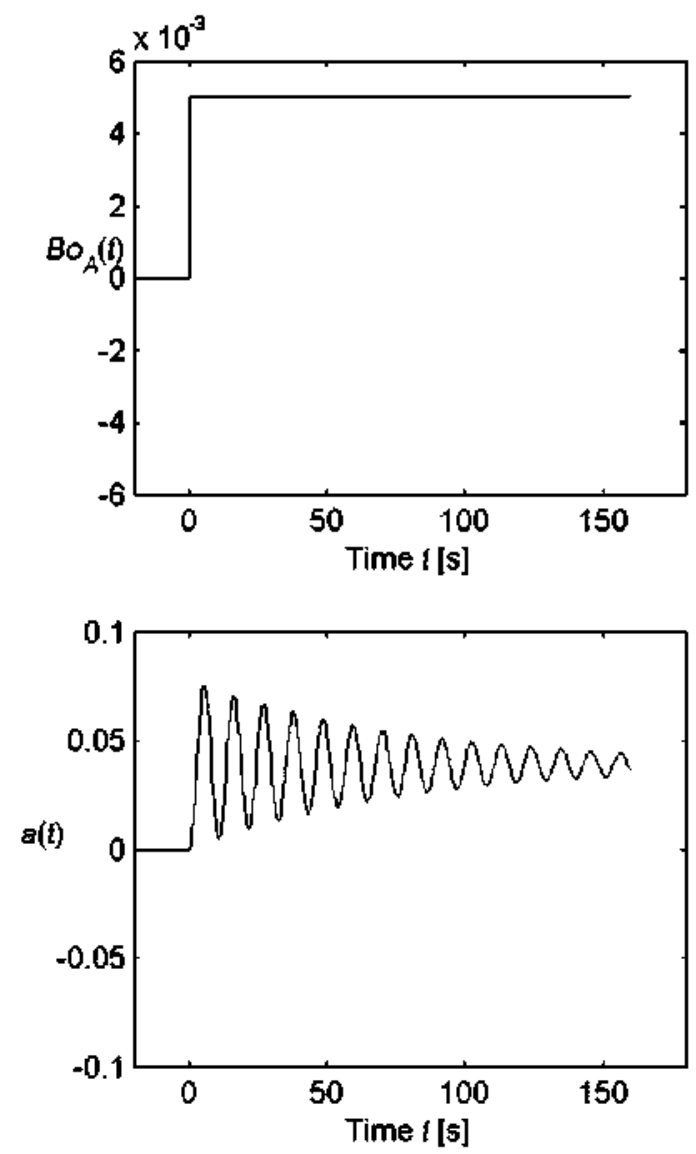

chosen to coincide with the average load deduced from the image sequence, and it is just intended to show the natural frequency and the damping characteristics, resulting in $T=10.7 \mathrm{~s}$ and a half-damping time of $t_{1 / 2} / t_{0}=2 \ln 2 / C=156$, or multiplying by the scale, $t_{1 / 2}=54 \mathrm{~s}$. The harmonic forcing amplitude and frequency, have been chosen to coincide with the apparent values found from image analysis, $B o_{\mathrm{A}}=0.001$ (found to match a typical radial deformation of $a=0.04$, or $0.6 \mathrm{~mm}$ against $14.5 \mathrm{~mm}$ ) and $T=12 \mathrm{~s}$.

Notice that, contrary to what was found in the analysis of Texus-33 experiment (Martinez et al., 2004), here, the non-linear effects are negligible (except close to the breaking), because the oscillations have an amplitude $a=0.04(0.6 \mathrm{~mm})$, so that non-linear effects only amount to (3/4) $a^{2} / \lambda<2 \%$, whereas in Texus-33 we had an amplitude of $a=0.2(6 \mathrm{~mm})$, so that non-linear effects amounted there to some $30 \%$.

\section{The experiment}

The experiment titled "Stability of long liquid columns", STACO for short, was carried out in Spacelab-D2 aboard Columbia (STS-55), with the investigators being the same as the authors of this paper.
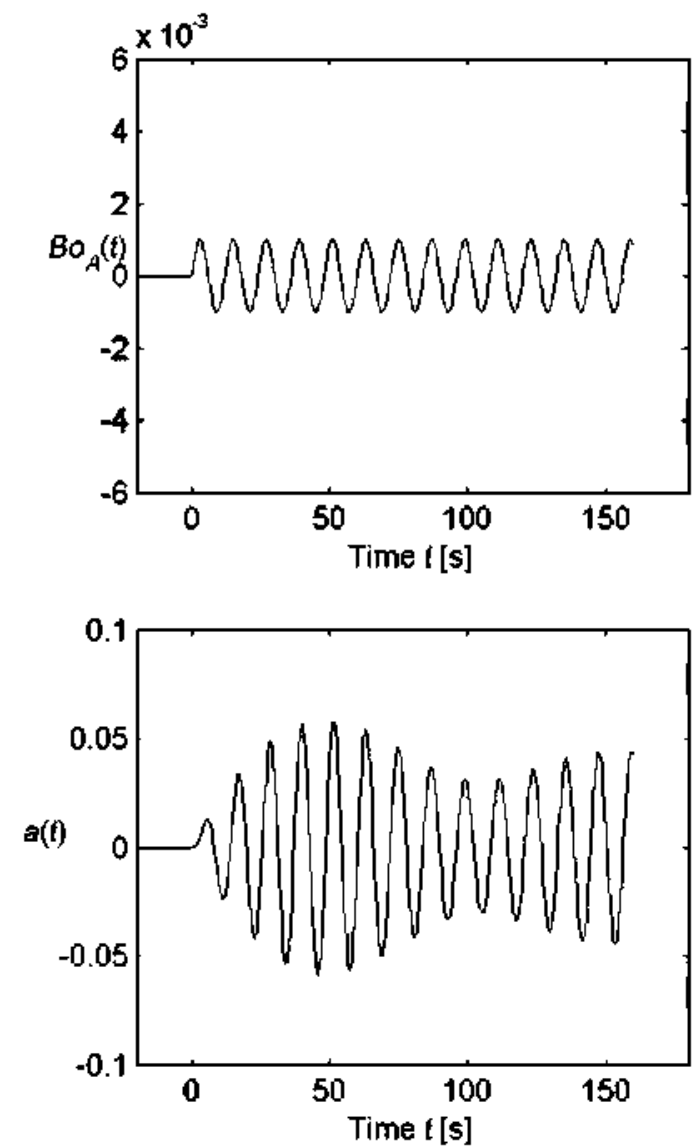

Fig. 4. Dynamic response of the liquid colımn to a step load and to a harmonic load. the latter close to the natural frequency of the system. 
STACO was executed in three runs with several days between each run. A top priority of the experiment was to reproduce previous Spacelab-D1 experiments of 1985 that were carried out in a similar facility (the first Fluid Physics Module, FPM, instead of the Advanced Fluid Physics Module, AFPM), at the same Spacelab location, with the same liquid configuration $(85 \mathrm{~mm}$ long column of $30 \mathrm{~mm}$ in diameter, instead of $100 \mathrm{~mm}$ long column of $35 \mathrm{~mm}$ in diameter), and using same materials and methods. Run 1 tested the response to forced oscillations imposed on one disc (at $0.40,0.41$ and $0.42 \mathrm{~Hz}$, around the column second eigenfrequency, predicted to be $0.41 \mathrm{~Hz}$ ), and it performed as predicted by theory. Run 2 tested the breakage of the column by slow separation of one disc at constant liquid volume, and it also performed as predicted. Run 3 was a bonus, and for the first time two unequal dises were used (this configuration is analysed in Bezdenejnykh and Meseguer (1991) and Perales et al. (1991)). With the sensitivity of the liquid column stretched to the limit, the liquid bridge broke apart, but in a non-understandable manner deserving further analysis. The basic data from liquid column experiments has always been the liquid outer shape recorded as an image sequence. The first and last video-frames are shown in Fig. 1.

The background grid is symmetric, formed by a central vertical line (behind the axis of the liquid column), with tick marks every $10 \mathrm{~mm}$ (uneven spaced in the images because of dioptical deflections), two vertical lines at $35 \mathrm{~mm}$ to each side of the axis (without tick marks), and two vertical strips of $10 \mathrm{~mm} \times 10 \mathrm{~mm}$ square cells centred at $50 \mathrm{~mm}$ to each side of the axis; everything drawn with $1 \mathrm{~mm}$ thick black lines on a blank paper, and transferred to a transparent plastic sheet.

The upper disc in Fig. 1, holding the liquid (and used to force vibrations of different amplitude and frequency on previous runs) is a $30 \mathrm{~mm}$ in diameter aluminium disc that has a $30^{\circ}$ cutback to a $27 \mathrm{~mm}$ in diameter stem, protruding $12 \mathrm{~mm}$ from a large base plate not seen in Fig. 1. Notice the small axial dimension of the cutback, of less than one millimetre: $((30-27) / 2) \tan 30^{\circ}=0.87 \mathrm{~mm}$.

The lower disc in Fig. 1 is a $28 \mathrm{~mm}$ in diameter aluminium dise that has a $30^{\circ}$ cutback to a $25 \mathrm{~mm}$ in diameter stem, protruding $6 \mathrm{~mm}$ from the large circular base plate seen in Fig. 1, where the parallax due to looking from just $800 \mathrm{~mm}$ apart, is apparent. The background grid is $80 \mathrm{~mm}$ further behind the axis, which means that the geometry at the liquid edge plane (the axial plane) is magnified by $880 / 800$ (a $110 \%$ magnification). The lower disc is used to feed the liquid from a motorised reservoir outside (through a $10 \mathrm{~mm}$ concentric hole in the disc), and can axially move up and down against the upper disc to establish the bridge initially and vary its slenderness. Both discs are also able to axially rotate at different rates (an iso-rotation trial was to start when the column broke).
The liquid used was a Dow Corning silicone oil of density $\rho=920 \pm 3 \mathrm{~kg} / \mathrm{m}^{3}$ at $25^{\circ} \mathrm{C}$, surface tension $\sigma=0.019 \pm 0.0005 \mathrm{~N} / \mathrm{m}$ and viscosity $\nu=5.0 \times 10^{-6}$ $\mathrm{m}^{2} / \mathrm{s}(5 \mathrm{cSt})$, and it was transparent.

Run 3 of STACO lasted more than half an hour from start to end, but we concentrate here just on the nearly 3 min that span from the precise moment the liquid column was finally established at the nominal configuration of length and volume (GMT $=125 / 14: 15: 35$ or Mission Elapsed Time 8/23:25:35), until the precise moment that the liquid column broke down, in an unrecoverable manner that prevented any further trials due to time limitation (GMT $=125 / 14: 18: 14$ or Mission Elapsed Time $8 / 23: 28: 14$ ); that is $160 \mathrm{~s}$, from where 4000 images have been extracted (at 25 frames per second) from the digitised videotape; more precisely, disruption took place $159.1 \pm 0.1 \mathrm{~s}$ after reaching the nominal configuration. The event happened to take place during a live video transmission from air to ground, and the coordinator on ground voiced the oncoming disruption $3 \mathrm{~s}$ before actual breakage.

There are minor modifications on the actual experiment data published in Martínez et al. (1995) and those here used, attributable to the conflicting data supplied by several instruments. First, in spite of the $65.6 \mathrm{~cm}^{3}$ of liquid fed according to data acquisition from the injection mechanism, a value of $V=63.2 \mathrm{~cm}^{3}$ is here used, based on volume computation from the images, $\boldsymbol{V}=63.2 \pm 0.5 \mathrm{~cm}^{3}$. Second, in spite of the $L=84.0 \mathrm{~mm}$ according to data acquisition from the disc-displacement mechanism, a value of $L=85 \mathrm{~mm}$ is here assumed, based on image analysis (a value of $L=86 \pm 1.5 \mathrm{~mm}$ is obtained by comparison with disc sizes and the aspect-ratio from the background squares); to reinforce that selection, there is the correlation with liquid volume, and that nothing else indicates that the operator did not reached the nominal value of $L=85 \mathrm{~mm}$. In short, it is impossible that a $84 \mathrm{~mm}$ long column of $65.6 \mathrm{~cm}^{3}$ yield the recorded shapes, and images are more reliable than potentiometers. With the present digitisation (Fig. 1), $100 \mathrm{~mm}$ from ten vertical squares in the background grid correspond to $503.5 \pm 0.5$ vertical pixels, whereas $100 \mathrm{~mm}$ horizontally between corresponding edges of the square strips measure $460 \pm 0.5$ horizontal pixels; at the column axis, the liquid column occupies $466 \pm 2$ vertical pixels, and the statistical value of disc radii are $74.2 \pm 0.2$ horizontal pixels and $68.8 \pm 0.2$ horizontal pixels. A small slant of some $0.1 \%$ can be deduced from the images, and attributed to the cumbersome optical path (two mirrors in between). The large uncertainty in column length from the images is due to the minute dove-tail cut-backs in the discs, hardly distinguished in Fig. 1, and the lack of any hard vertical reference in the moving parts (the large upper end-plate is out of view in Fig. 1). Finally, the settings for disc oscillations that appeared in the 'as flown' 
data package $(1 \mathrm{~mm}$ amplitude and $0.1 \mathrm{~Hz}$ ), are not applicable since there are settings left from previous trials, but not executed, as the images clearly demonstrate (the upper disc would oscillate between six pixels!).

It is apparent from the video recording of the liquid column that there is a combined motion, with axial oscillations in an S-shape, and lateral oscillations in a C-shape. A rather comprehensive display of the actual evolution of the liquid column during these $160 \mathrm{~s}$ (as a substitute of the video clip, available on request), is presented in Fig. 5, were the radius at 5 selected equidistant slices of the column are presented versus time; the only manipulation being an overall scaling to match the actual dise sizes (some spurious spikes can be seen, corresponding to single-frame video synchrony failures).

One may see in Fig. 5, the raw discretisation uncertainty (the top and bottom radii) should be exactly 15 and $14 \mathrm{~mm}$ in diameter (better than a tenth of a millimetre according to manufacture). The radius at the middle of the column remains steady at some $16 \mathrm{~mm}$ of value, greater than the disc radii, showing that the motion of the liquid outline has a node midway between the dises, and that the volume of liquid is larger than the mid cylinder between the discs. The two radii at $1 / 4$ and $3 / 4$ of the column length, oscillate in counter-phase (maintaining the volume) with a period of $12.0 \pm 0.2 \mathrm{~s}$ and an amplitude (in the 5 almost equal cycles to the right in Fig. 5) of $1 \pm 0.1 \mathrm{~mm}$ peak to peak.

\section{Results}

The results here presented are based on the analysis of the onboard-recorded VHS video tape, digitised to a $720 \times 576$ pixel map of 24 bit grey-scale, in a sequence of 4000 images at 25 images per second (the original video has 30 frames per second), and analysed with Mat$\mathrm{lab}^{(3)}$ software, that has been used to make all the computations and plots from the image sequence; in particular, the edges of the liquid bridge are automatically extracted using the Canny algorithm, which finds edges by looking for local maxima of the gradient of the grey-level matrix, using the derivative of a Gaussian filter. Two such images and their outlines (by automatic edge detection) are shown in Fig. 1.

From the image sequence, the following information has been extracted:

1. Length of the column, $L=86 \pm 1.5 \mathrm{~mm}$, by comparison with disc sizes of known size and the aspect-ratio from the background squares. This large uncertainty from the images has been reduced by knowing that the nominal setting was $85 \mathrm{~mm}$, as explained before in Section 2.

2. Volume of liquid, $V=63.2 \pm 0.5 \mathrm{~cm}^{3}$, by using

$\mathrm{V}=\int_{-L}^{L} \pi R^{2}(z) \mathrm{d} z=\pi \sum_{i=1}^{n} R_{i}^{2} \frac{2 L}{n}$

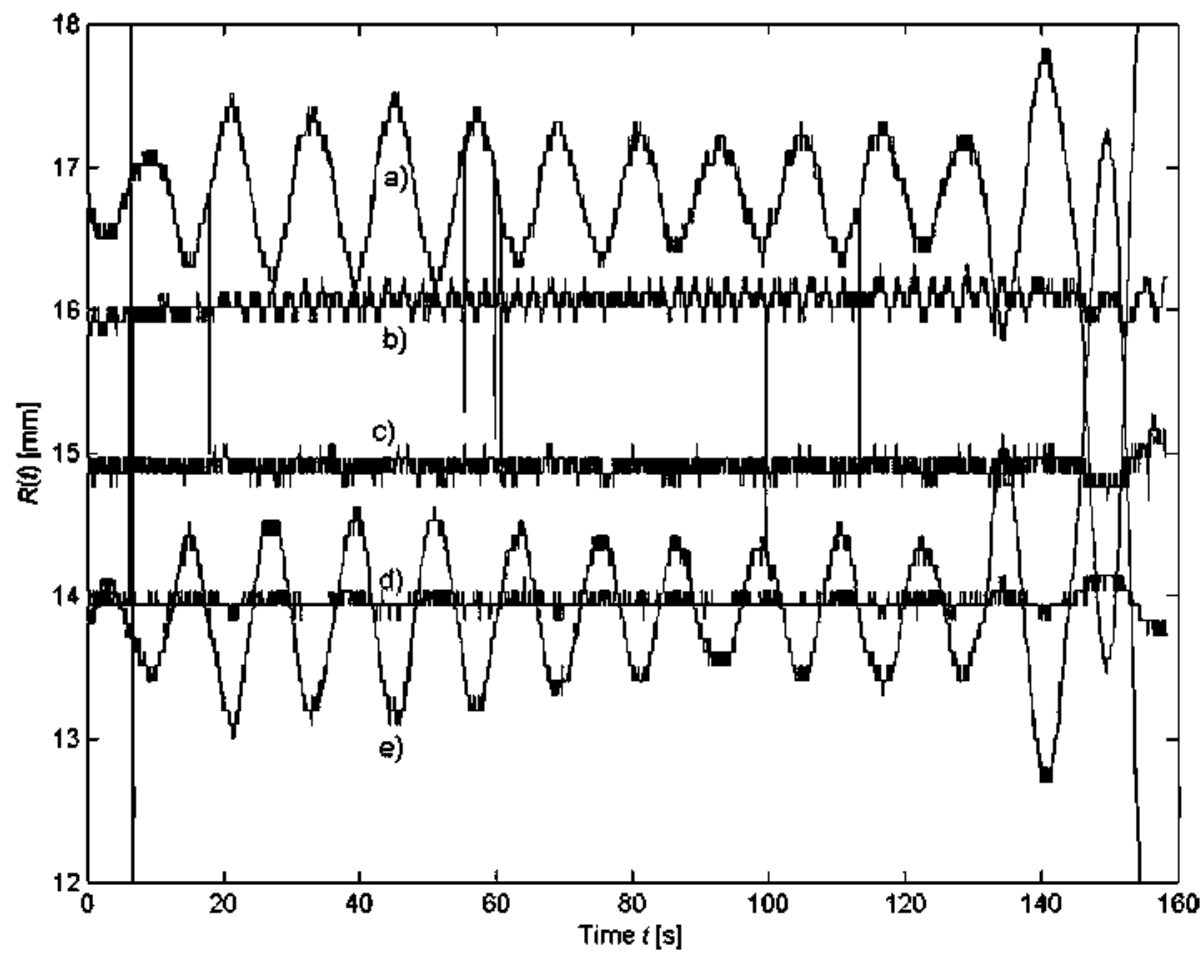

Fig. 5. Selected equidistant slice radii vs. time, for the $160 \mathrm{~s}$ of the experiment (between the two images in Fig. 1): (a) radius at $3 / 4$ of the column length, from botton (see Fig. 1); (b) at $2 / 4$ of the length: (c) at 4/4. i.e.. at the upper disc; (d) at the botton disc: (e) at $1 / 4$ of the colunn. The initial distortions are due to the transients remaining from the filling and stretching of the liquid column. 
where $R_{i}$ are the radii of the discretised slices in the column and $n$ the number of pixel lines from one disc to the other (466 in our case). The uncertainty is small, as can be seen from the evolution of the computed volume with time, $V(t)$, in Fig. 6 , but several details show up: (1) there is a tendency for the volume to increase a little bit, maybe due to some resilience in the filling mechanism; (2) there is a clear increase in the uncertainty some $50 \mathrm{~s}$ before the column broke $(t=110 \mathrm{~s}$ in Fig. 6); (3) there is a rippling at precisely the first natural frequency of the lateral dynamics, surely related to the artificial circularisation of the off-axis column slices; (4) the rippling is biased towards larger volumes (yet unexplained).

3. Lateral motion, by using

$$
r(z, \theta, t)=r_{\mathrm{eq}}(z)+c(t) \frac{\left(\Lambda^{2}-z^{2}\right)}{2} \cos \theta
$$

and finding the amplitude evolution $c(t)$ by fitting the slice centre line. The liquid column appears to oscillate laterally in agreement with results from Sanz and López Díez (1989), with a period of $T_{\mathrm{lat}}=$
$4.8 \pm 0.2 \mathrm{~s}$ and an amplitude of less than $2 \mathrm{~mm}$ at the antinode (midway between discs), without any major influence of the breaking process. Notice how perfect the matching of experimental shapes to the eigenfunction $\left(A^{2}-z^{2}\right)$ happens to be, because the shape residue, defined as the root mean square of all digitised deformations relative to the best-fit eigenfunction, has a mean white noise value of $<1 \%$ (imperceptible at the scale in Fig. 6).

4. Axial motion, by using (3). The liquid column appears to oscillate axially as modelled in Eq. (3), with a period of $T_{\mathrm{lat}}=12 \pm 0.2 \mathrm{~s}$ and an amplitude of $0.6 \mathrm{~mm}$ at the antinode (at $1 / 4$ and $3 / 4$ of the column length), most of the time; but, at around $t=130 \mathrm{~s}$ in Fig. 6, the amplitude of the oscillation starts to grow until surpassing the stability limits (computed from Eq. (4) to be at $a \pm 0.24$, or in dimensional units $\pm 3.5 \mathrm{~mm}$ ), and breaking with the larger drop formed spreading over the dise edge onto the base plate and ending the experiment. Notice how perfect the matching of experimental shapes to the model of Eq. (3) happens to be here
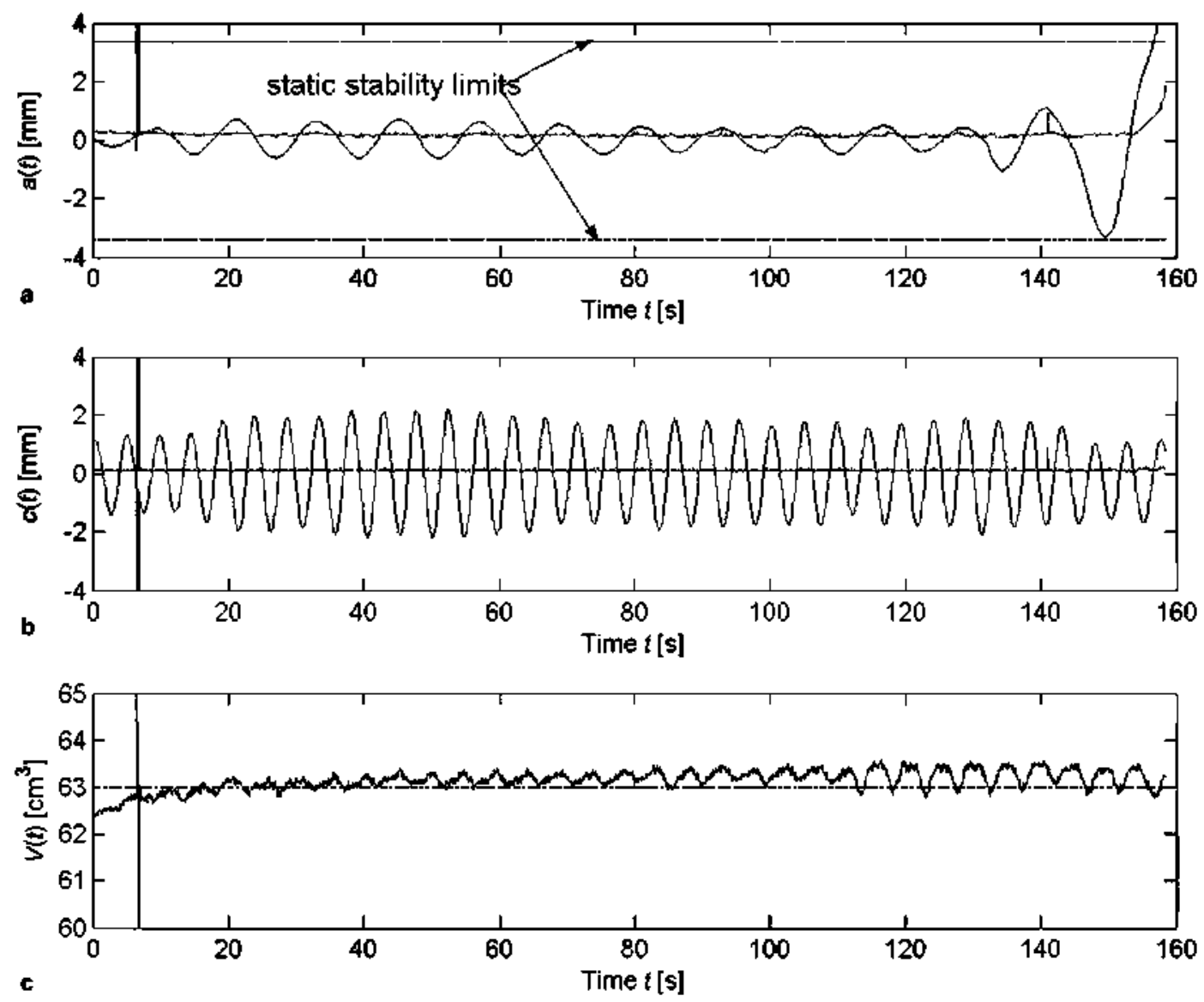

Fig. 6. Analysis of the liquid column evolution during the experiment (disruption occurs at $159.1 \mathrm{~s}$ ). (a) Axial dynamics: the wavy line is the best-fit amplitude $a(t)$. defined by Eq. (3), the shape residue is nearly constant (except at the end), and the static stability bounds are shown labelled. (b) Lateral dynamics: the wavy line is the best-fit amplitude $c(t)$. defined by Eq. (6). the shape residue is near constant. (c) Volume of liquid. from Eq. (5). and representative value. All in dimensional units. There is a video-synchronism flaw apparent near $t=7 \mathrm{~s}$. 
too, since the mean shape residue is $<1 \%$ too (imperceptible at the scale in Fig. 6), but this time with a minor beating at the same frequency.

5. Apparent axial acceleration. If we compute the average shape during the 9 cycles of quasi-periodic oscillation (those later shown in Fig. 5), we find that it corresponds pretty well with the static equilibrium shape for $B o_{\mathrm{A}}=0.005 \pm 0.0002(50 \pm 2 \mu \mathrm{g})$, and the maximum and minimum dynamic shape deformation correspond to $B o_{\mathrm{A}}=0.002 \pm 0.0005$ and $B o_{\mathrm{A}}=$ $0.008 \pm 0.0005$, as presented in Fig. 3, where other relevant liquid column equilibrium shapes have been plotted. Surprisingly enough, it appears that the apparent residual g-level depends on the actual motion the liquid has (it was $5 \pm 2 \mu \mathrm{g}$ from the analysis of run 1 of STACO).

In summary, it follows from the analysis that the liquid column shows an unexplained dynamical behaviour, in spite of being intentionally left idle, waiting for the initial disturbances to die out. This deviation can be quantified in the following details:

- The liquid column oscillates for some $120 \mathrm{~s}$ without signs of viscous damping (against the expected halfdamping time of $54 \mathrm{~s}$ ). One must conclude that there is a contribution from ambient noise at least to match the viscous term in Eq. (4), $B o_{\mathrm{A}}(t)=m C \mathrm{~d} a(t) / \mathrm{d} t$ of amplitude $B o_{\mathrm{A}}=0.0005(5 \mu \mathrm{g})$ at the first axial eigenfrequency (lateral dynamics is not yet developed so far).

- The mean shape (the $120 \mathrm{~s}$ average) has axial symmetry. One must conclude that there is no residual lateral acceleration.

- The mean shape does not correspond to an unloaded equilibrium shape; it shows a residual amphora-type deformation $0.2 \mathrm{~mm}$ above the unloaded equilibrium shape, as if corresponding to a liquid column with a residual axial acceleration of $B O_{\mathrm{A}}=0.005(50 \mu \mathrm{g})$. Where does this permanent residual load come from? Is it a second-order effect of the oscillations, a kind of streaming?

- A quasi-steady axial oscillation with a frequency similar to the predicted natural frequency (a period of $12 \mathrm{~s})$ and a $0.5 \mathrm{~mm}$ amplitude is superposed to the mean shape. Where is the excitation to sustain this motion?

- A quasi-steady lateral oscillation with a frequency similar to the predicted natural frequency (a period of $5 \mathrm{~s}$ ) and a very large amplitude of $2 \mathrm{~mm}$ is superposed to the mean shape. Where is the excitation maintaining this motion?

- After 11 sustained cycles, the axial deformation gently swings divergently until the liquid bridge disrupts 2 cycles after. Where the perturbations come from? Was it a stochastic resonance effect?
- A more viscous liquid would have responded less widely to the uncontrolled dynamical stimuli, of course, but at this stage in research we wanted maximum sensitivity.

\section{Conclusions}

We have made here a detailed analysis of a unique experiment where an enigmatic event took place: a long liquid column between unequal discs of 28 and $30 \mathrm{~mm}$ in diameter was gently oscillating during more than $120 \mathrm{~s}$, both axially and laterally, much more than expected from ambient disturbances according to previous trials, when finally the axial swing grew until breaking the bridge in some additional $30 \mathrm{~s}$.

We have found that long liquid columns are very sensitive to disturbances, dynamic effects growing when sensitivity increases, and, although theories are available to analyse in detail the liquid response, we have not yet been able to ascertain the stimuli responsible for the present breakage.

Automated image analysis has progressed a lot, but small problems remain. Here, we benefit from overall image analysis, unfeasible in the past, but details of the disc edges cannot be reconstructed from the old videotapes. Digital imaging nowadays would solve many of the old video problems.

A possible explanation to the fact that the liquid column appears more slender in the images than in reality (after thorough calibration with the background reference grid), may be that images of the liquid column give always a smaller value for radii due to reflection of the grazing rays.

A simplistic conclusion might be that the experiment went wrong because of lack of proper control: some fortuitous push broke the column and that is all one may say, but this is not a valid explanation for the following reasons:

- Efforts had been taken to avoid pushes (there was 'microgravity period' in the timeline).

- Efforts had been taken to record any fortuitous event and there is no event in the acceleration records.

- The theory describing liquid column behaviour had been developed a lot to understand any possible response (although not fully, as evidenced here). Theoretical simulations show, as it can be expected, that a single-event or step load cannot account for this several-period divergence (the breakage would had been in less than $5 \mathrm{~s}$ instead of $30 \mathrm{~s}$ ).

A characteristic of experimentation is reproducibility and this experiment has not been reproduced yet. We believe that the challenging behaviour of this 
unequal-disc liquid column, which provides the only such data available at the time, merits further experimental work.

\section{References}

Bezdenejnykh. N.A., Meseguer, J, Stability linits of minimum volume and breaking of axisymmetric liquid bridges between unequal disks. Microgravity Science and Technology 4, 235-239. 1991.

Martínez, I., Perales. J.M. Mechanical behaviour of liquid bridges in microgravity. in: Monti. R. (Ed.). Physics of Fluids in Microgravity. Taylor \& Francis, London, pp. 21-45, 2001.

Martinez, I., Perales. J.M.. Gomez. M. Effects of axial and centrifugal forces on the stability of liquid bridges. ESA SP-333. pp. 123-130, 1992.
Martinez. I., Perales, J.M. Meseguer. J. Non-linear response of a liquid bridge to a sinusoidal acceleration under microgravity. Experiments in Fluids 37, 775-781, 2004.

Martínez, I., Perales, J.M., Meseguer. J. Stability of long liquid columns (SL-D2- FPM-STACO), in: Sahm. P.R., Keller, M.H., Schiewe, B. (Eds.), Scientific Results of the German Spacelab Mission D-2. WPF, pp. 220-231, 1995.

Meseguer, J., Bezdenejnykh, N.A. Rodriguez de Francisco. P. On the use of liquid bridges as accelerometers. Microgravity Science and Technology IX/2, 62-69, 1996.

Meseguer. J., González, M.A.. Alexander, J.I.D. Dynamic stability of long, axisynmetric liquid bridges. Microgravity Science and Technology 7. 246-251, 1994.

Perales. J.M., Meseguer, J., Martinez, I. Minimum volume stability linits for axisynmetric liquid bridges subject to steady axial acceleration. Journal of Crystal Growth 110, 855-861, 1991.

Sanz. A., López Diez, J. Non-axisymmetric oscillations of liquid bridges. Journal of Fluids and Mechanics 205, 503-521, 1989. 